\title{
PREPARATION, CHARACTERIZATION AND EVALUATION OF FLOATING MICROPARTICLES OF CIPROFLOXACIN
}

\author{
SUMIT DURGAPAL ${ }^{* 1}$, SAYANTAN MUKHOPADHYAY ${ }^{2}$, LAXMI GOSWAMI ${ }^{3}$
}

${ }^{1}$ Department of Pharmaceutical Sciences, Bhimtal Campus, Kumaun University Nainital, Uttrakhand India, ${ }^{2}$ Department of Pharmaceutical Sciences, SGRRITS, Patel Nagar, Dehradun, Uttrakhand, India, ${ }^{3}$ Government Polytechnic Mallital, Nainital Uttrakhand India

Email: sumit.1459@gmail.com

Received: 19 Jul 2016, Revised and Accepted: 02 Dec 2016

\section{ABSTRACT}

Objective: The main purpose of this study is to prepare a floating micro articulated drug delivery system of ciprofloxacin by using non-aqueous solvent evaporation technique to increase the bioavailability and therapeutic effectiveness of the drug by prolonging its gastric residence time.

Methods: Floating microparticles were prepared by using different low-density polymers such as ethyl cellulose and hydroxypropyl methylcellulose either alone or in combination with the aid of non-aqueous solvent evaporation technique. All the formulated microparticles were subjected to various evaluation parameters such as percentage yield, drug content, drug entrapment, rheological studies, floating characteristics and in vitro drug release studies.

Results: Drug-excipient compatibility studies performed with the help of FTIR instrument indicated that there were no interactions. Results revealed that non-aqueous solvent evaporation technique is a suitable technique for the preparation of floating microspheres as most of the formulations were discrete and spherical in shape with a good yield of $65 \%$ to $85 \%$ and 15 to $22 \mathrm{~h}$ of floating duration with $90 \%$ of maximum percentage floating capacity shown by formulation $\mathrm{FM}_{9}$. Though, different drug-polymer ratios, as well as a combination of polymers, play a significant role in the variation of overall characteristics of formulations. Based on the data of various evaluation parameters such as particle size analysis, drug content, drug entrapment, rheological studies and in vitro drug release characteristics formulation $\mathrm{FM}_{9}$ was found to fulfil the criteria of ideal floating drug delivery system.

Conclusion: Floating microparticles were successfully prepared, and from this study, it can be concluded that the developed floating microspheres of ciprofloxacin can be used for prolonged drug release in the stomach to improve the bioavailability and patient compliance.

Keywords: Floating Microparticles, Ciprofloxacin, Ethylcellulose and HPMC.

(C) 2016 The Authors. Published by Innovare Academic Sciences Pvt Ltd. This is an open access article under the CC BY license (http://creativecommons.org/licenses/by/4.0/) DOI: http://dx.doi.org/10.22159/ijap.2017v9i1.14183

\section{INTRODUCTION}

Oral controlled release (CR) dosage forms (DFs) have been developed for the past 3 decades due to their considerable therapeutic advantages. However, this approach has not been suitable for a variety of important drugs, characterised by a narrow absorption window in the upper part of the gastrointestinal tract i.e. stomach and small intestine. This is due to the relatively short transit time of the DF in these anatomical segments. Thus, after only a short period of less than $6 \mathrm{~h}$, the CR-DF has already left the upper gastrointestinal tract, and the drug is released in non-absorbing distal segments of the gastrointestinal tract. This results in a short absorption phase that is often accompanied by lesser bioavailability. The medications that are included in the category of narrow absorption window drugs are mostly associated with improved absorption at the jejunum and ileum due to their enhanced absorption properties e. g. large surface area, in comparison to the colon; or because of the enhanced solubility of the drug in the stomach as opposed to more distal parts of the gastrointestinal tract. It was suggested that compounding narrow absorption window drugs in a unique pharmaceutical DF with gastro retentive properties would enable an extended absorption phase of these drugs. After oral administration, such a DF would be retained in the stomach and release the drug there in a controlled and prolonged manner, so that the drug could be supplied continuously to its absorption sites in the upper gastrointestinal tract. This mode of administration would best achieve the known pharmacokinetic and pharmacodynamics advantages of CR-DFs for these drugs. The need for gastro-retentive dosage forms (GRDFs) has led to extensive efforts in both academia and industry towards the development of such drug delivery systems. These efforts resulted in GRDFs that were designed in large part [1].

Over the past three decades, the pursuit and exploration of devices designed to be retained in the upper part of the gastrointestinal (GI) tract has advanced consistently in terms of technology and diversity, encompassing a variety of systems and devices such as floating systems, raft systems, expanding systems, swelling systems, bioadhesive systems and low-density systems. Gastric retention will provide advantages such as the delivery of drugs with narrow absorption windows in the small intestinal region. Also, longer residence time in the stomach could be advantageous for local action in the upper part of the small intestine, for example, treatment of peptic ulcer disease. Furthermore, improved bioavailability is expected for drugs that are absorbed readily upon release in the GI tract. In the present study, the floating micro particles of ciprofloxacin as GRDFs were prepared using polymers such as ethyl cellulose and HPMC K4M at different proportions using individual polymer or in a combination of both polymers with the help of nonaqueous solvent evaporation technique [2].

\section{MATERIALS AND METHODS}

Ciprofloxacin was obtained as a gift sample from Zydus Cadila, Baghekhola, E. Sikkim. Polymers such as hydroxypropyl methylcellulose (HPMC) K4M was obtained from Loba-Chem. Pvt. Ltd., Mumbai. Ethyl cellulose was provided by Thomas Baker Pvt. Ltd., Mumbai. All other reagents and chemicals were of analytical grade.

\section{Drug-polymer compatibility study through FTIR}

Before the preparation of microspheres, compatibility of the drug with polymers present in microspheres was performed. The Fourier transform infrared radiation measurement (FTIR) spectral measurements were taken at ambient temperature using IR spectrophotometer (Shimadzu, model 840, Japan). In this method peak of the pure drug was matched with that of drug and polymer in the range of $4000-400 \mathrm{~cm}^{-1}$ for 100 scans [3].

Preparation of microspheres by non-aqueous solvent evaporation method

Microspheres containing antibiotic drug ciprofloxacin were prepared by a non-aqueous solvent evaporation method. Briefly, drug and ethyl 
cellulose were mixed in acetone at various ratios. The slurry was slowly introduced into $30 \mathrm{ml}$ of liquid paraffin while being stirred at $1200 \mathrm{rpm}$ by a mechanical stirrer equipped with a three-bladed propeller at room temperature. The solution was stirred for $4 \mathrm{hr}$ to allow the solvent to evaporate completely and the microspheres were collected by filtration. The microspheres were repeatedly washed with petroleum ether $\left(40^{\circ}-60^{\circ} \mathrm{C}\right)$ until free from oil. The collected microspheres were dried for $1 \mathrm{~h}$ at room temperature and subsequently stored in desiccators over fused Calcium chloride [4]. Compositions of different formulations are given in table 1,2 and 3.

Table 1: Formulation composition of microspheres prepared by using ethyl cellulose

\begin{tabular}{lllll}
\hline S. No. & Ingredients & Quantity & \\
\cline { 2 - 5 } & & FM $_{\mathbf{1}}$ & FM $_{\mathbf{2}}$ & FM $_{\mathbf{3}}$ \\
\hline 1 & Ciprofloxacin $(\mathrm{mg})$ & 100 & 100 & 100 \\
2 & Ethyl Cellulose(mg) & 100 & 200 & 100 \\
3 & Acetone(ml) & 12 & 400 & 12 \\
\hline
\end{tabular}

Table 2: Formulation composition of microspheres prepared by using HPMC

\begin{tabular}{lllll}
\hline S. No. & Ingredients & Quantity & & \\
\cline { 2 - 5 } & & FM $_{\mathbf{5}}$ & FM $_{\mathbf{6}}$ & FM $_{\mathbf{7}}$ \\
\hline 1 & Ciprofloxacin $(\mathrm{mg})$ & 100 & 100 & 100 \\
2 & HPMC(mg) & 100 & 200 & 300 \\
3 & Acetone(ml) & 12 & 400 & 12 \\
\hline
\end{tabular}

Table 3: Formulation composition of microspheres prepared by using combination of ethyl cellulose and HPMC

\begin{tabular}{lllll}
\hline S. No. & Ingredients & Quantity & \\
\cline { 2 - 5 } & & FM9 & FM $_{\mathbf{1 0}}$ & \\
\hline 1 & Ciprofloxacin (mg) & 100 & 100 & FM $_{\mathbf{1 1}}$ \\
2 & Ethyl cellulose (mg) & 100 & 100 & 200 \\
3 & HPMC (mg) & 100 & 200 & 100 \\
4 & Acetone (ml) & 12 & 12 & 12 \\
\hline
\end{tabular}

FM = Formulation Microsphere, HPMC $=$ Hydroxypropyl methylcellulose

\section{Evaluation of prepared floating microspheres}

\section{Determination of yield of microspheres}

\section{Determination of \% yield of microspheres}

Thoroughly dried microspheres were collected and weighed accurately. The percentage yield was then calculated using formulae given below [5].

$$
\text { Percentage Yield }=\frac{\text { Weight of obtained microspheres }}{\text { Total weight of drug and polymer }} \times 100
$$

\section{Determination of particle size of microspheres}

The particle size of prepared microspheres from each batch was determined by the optical microscopy method using an ocular micrometre which was previously calibrated with stage micrometre [5].

\section{Measurement of floating capacity}

An in vitro floating study was carried out using 0.1(N) HCL as a dispersing medium. Microspheres were spread over the surface of $400 \mathrm{ml}$ of dispersing medium at $37 \pm 0.5^{\circ} \mathrm{C}$. A paddle rotating at 100 $\mathrm{rpm}$ agitated the medium. Each fraction of microspheres floating on the surface and those settled down were collected at a predetermined time point. The collected samples were weighed after drying [6].

Percentage of floating of microspheres

$$
=\frac{\text { weight of floating microspheres }}{\text { Initial weight of microspheres }} \times 100
$$

\section{Determination of drug content in microspheres}

Drug-loaded microspheres (100 mg) were powdered and suspended in $100 \mathrm{ml} 0.1(\mathrm{~N}) \mathrm{HCL}$ solutions and kept for $24 \mathrm{~h}$. It was stirred for 5 min and filtered. Ciprofloxacin content in the filtrate was determined spectrophotometrically at $278 \mathrm{~nm}$ using a regression derived from the standard curve [5].

\section{Determination of drug entrapment in microspheres}

The drug entrapment efficiency was calculated by the equation [5].

$$
\text { Entrapment Efficiency }=\frac{\text { Practical Drug Content }}{\text { Theoritical drug content }} \times 100
$$

The entire test was performed in triplicate.

\section{Rheological properties}

The angle of repose, Carr's index, Bulk density, True density, Porosity, and Hausner's ratio were determined to assess the flowability of the prepared microspheres.

\section{Angle of repose}

The angle of repose was determined by using funnel method; the accurately weighed spheres were taken in the funnel. The height of funnel was adjusted in such as way that the tip of funnel just touches the apex of the heap of blends. The blends were allowed to flow through funnel freely onto the surface. The diameter of powder cone was measured; angle of repose was calculated by using following equation [7].

$$
\operatorname{Tan} \theta=\frac{\mathrm{h}}{\mathrm{r}}
$$

Where the; h-height of pile, $\theta$-angle of repose, $r$-radius of base pile and $<25$-excellent flow, 25-30-good flow, 30-40-passable, $>40$-very poor flow.

\section{Bulk density}

Apparent bulk density $\left(\rho_{b}\right)$ was measured by pouring the pre-weight (M) blend into a graduated cylinder. The bulk volume $\left(\mathrm{V}_{\mathrm{b}}\right)$ of the blend was determined. Then the bulk density was calculated by using the formula [7-9]. 


$$
\rho b=\frac{M}{V b}
$$

\section{Tapped density}

The measuring cylinder containing a known mass (M) of the blend was tapped for a fixed time, and the minimum volume $\left(\mathrm{V}_{\mathrm{t}}\right)$ occupied in the cylinder was measured. The tapped density $\left(\rho_{t}\right)$ was calculated by using the following formula [7-9].

$$
\rho t=\frac{M}{V t}
$$

\section{True density}

True density is defined as the ratio of the weight of powder and the tapped volume of powder. It was determined by placing a graduated cylinder, containing a known mass of microspheres. The cylinder was allowed to fall under its own weight onto a hard surface from the height of $10 \mathrm{~cm}$ at a 2 -second interval. The tapping was continued until no further change in volume was noted [8-10].

True density $(\rho p)=\frac{\text { Mass of microspheres }}{\text { Volume of microspheres after tapping }(\mathrm{Vp})} \times 100$

\section{Consolidation index \%}

It is one of a method for determining flow properties and also called as Carrs's index of compressibility. It is indirectly related to the relative flow rate, cohesiveness and particle size. It is a simple, fast and popular method of predicting powder flow characteristics $[8,9]$.

Percentage Consolidation index

$$
=\frac{\text { Tapped density }- \text { Bulk density }}{\text { Tapped density }} \times 100
$$

In Carr's index, the value below $15 \%$ indicates a powder with usually give rise to good flow characteristics, whereas above $25 \%$ indicates poor flowability. Lower Hausner's ratio $(<1.25)$ indicates better flow properties than higher ones ( $>1.25)$

Hausner's ratio

$$
\text { Hausner's ratio }=\frac{\text { Tapped Density }}{\text { Bulk Density }} \times 100
$$

Lower Hausner's ratio $(<1.25)$ indicates better flow properties than higher ones $(>1.25)[8,9]$.

\section{Loose surface crystals study}

Ciprofloxacin loaded microspheres were evaluated for loose surface crystal study to observe the excess drug present on the surface of microspheres. From each batch, $100 \mathrm{mg}$ microsphere formulation was accurately weighed and transferred carefully to the $100 \mathrm{ml}$ of double distilled water. After vigorous shaking for 5 min, the system was subjected to filtration through Whatman filter paper 41. The amount of drug lost infiltrate was determined spectrophotometrically and calculated as a percentage of total drug content [11].

\section{Determination of swelling properties}

The dynamic swelling property of microspheres in the dissolution medium was determined. Microspheres of known weight were placed in dissolution solution for $3 \mathrm{hr}$ and the swollen microspheres were collected by a centrifuge and the wet weight of the swollen microspheres was determined by first blotting the particles with filter paper to remove absorbed water on the surface and then weighed immediately on an electronic balance [12]. The percentage of swelling of microspheres in the dissolution media was then calculated by using following equation-

$$
\mathrm{Sw}=\frac{\text { WFinal }- \text { Winitial }}{\text { Winitial }} \times 100
$$

\section{Scanning electron microscopy (SEM)}

Scanning electron microscopy was carried out to study the morphological characteristics of ciprofloxacin microspheres. The dried microspheres were coated with gold $\left(100 \mathrm{~A}^{\circ}\right)$ under an argon atmosphere in a gold coating unit and Scanning electron micrographs were observed [13].

\section{In vitro drug release study}

USP-II type dissolution apparatus (paddle type) was performed at $50 \mathrm{rpm}$ in $900 \mathrm{ml} 0.1 \mathrm{~N} \mathrm{HCL} .5 \mathrm{ml}$ of sample was withdrawn at a predetermined interval, and the volume of dissolution medium was maintained by adding the same volume of dissolution medium to maintain the sink condition. The absorption of withdrawn sample was measured spectrophotometrically with suitable dilution and the corresponding concentration was determined from the respective calibration curve. The temperature was maintained at $37{ }^{\circ} \mathrm{C}$ throughout the studies [14, 15].

\section{In vitro drug release kinetics}

Kinetic models are best-known tools to describe the drug release pattern from immediate and modified release dosage forms. In order to investigate the kinetics and mechanism of drug release from prepared microspheres of different drug and polymers ratios, the release data were examined using Zero order kinetic, First order kinetic, Higuchi kinetic, Hixon-Crowell and Korsmeyer-Peppas model [16].

\section{RESULTS AND DISCUSSION}

Floating microspheres of ciprofloxacin were prepared using polymers such as ethyl cellulose and hydroxypropyl methyl cellulose either alone or in combination in various drug and polymer ratios as given in formulation composition of microspheres (table 1,2 and 3) with the help of non-aqueous solvent evaporation technique. Formulations FM1 to FM4 were prepared using gradually increasing the concentration of ethyl cellulose and formulations FM5 to FM8 were prepared using HPMC with fixed drug concentration of $100 \mathrm{mg}$. For the formulations of FM9 to FM12 combination strategy of polymers such as ethyl cellulose and hydroxypropyl methylcellulose (HPMC) was used. In these formulations, the amount of drug was kept constant with varying amount of both the polymers with respect to each other.

All the microspheres obtained from non-aqueous solvent evaporation technique were discrete and spherical in shape. The optical microscopic data of each formulation is given in table 4. Scanning electron microscopy (SEM, fig. 2) was done to determine the surface microscopy and internal texture of microspheres. The size of all the microspheres was found to be in the range $100-200 \mu \mathrm{m}$ and increases with the increase in polymer concentration. Surface topology report (fig. 2) confirms the particle size and provides evidence about the smooth surface of prepared microsphere with no fracture.

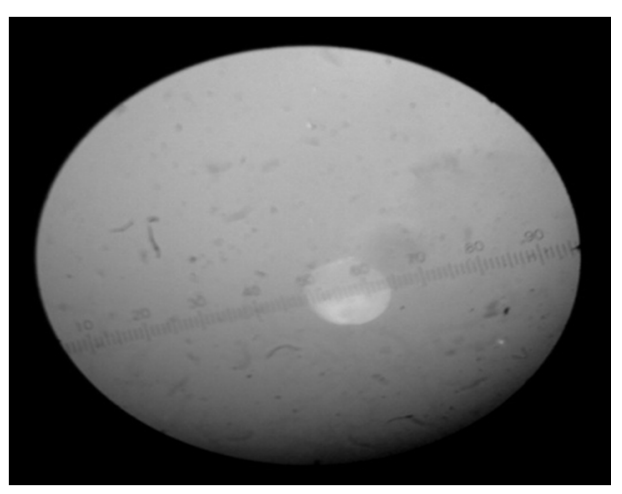

Fig. 1: Microscopic view of formulation FM9

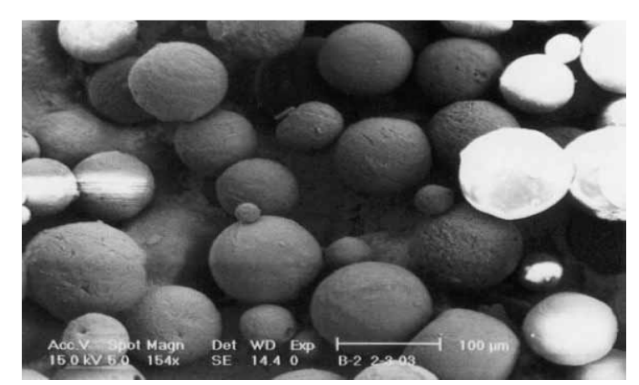

Fig. 2: Scanning electron photomicrograph of formulation FM9 
Table 4: Particle size analysis of microspheres

\begin{tabular}{llll}
\hline Batch code & Particle size $(\boldsymbol{\mu m})$ mean \pm SD $(\mathbf{n}=\mathbf{3})$ & Batch code & Particle size $(\boldsymbol{\mu m})$ mean \pm SD $(\mathbf{n}=\mathbf{3})$ \\
\hline $\mathrm{FM}_{1}$ & $135.5 \pm 2.33$ & $\mathrm{FM}_{2}$ & $158.2 \pm 2.31$ \\
$\mathrm{FM}_{3}$ & $175.3 \pm 2.71$ & $\mathrm{FM}_{4}$ & $198.7 \pm 1.29$ \\
$\mathrm{FM}_{5}$ & $145.5 \pm 2.92$ & $\mathrm{FM}_{6}$ & $160.7 \pm 1.43$ \\
$\mathrm{FM}_{7}$ & $175.9 \pm 1.49$ & $\mathrm{FM}_{8}$ & $195.7 \pm 2.12$ \\
$\mathrm{FM}_{9}$ & $120.5 \pm 3.56$ & $\mathrm{FM}_{10}$ & $145.1 \pm 3.52$ \\
$\mathrm{FM}_{11}$ & $175.7 \pm 2.17$ & $\mathrm{FM}_{12}$ & $148.7 \pm 3.81$ \\
\hline
\end{tabular}

Percentage yield of all the formulations (table 5) prepared from nonaqueous solvent evaporation technique was found in the range $66-85 \%$ which is sufficiently high. As experimental result revealed \% yield value was directly related with polymeric concentration. In most of the cases as the polymer concentration increase, \% yield was also increased.

The success of floating type of gastro retentive systems depends greatly on the parameters such as duration of floating and percentage of floating capacity, because as long as the microspheres remain buoyant on the surface of gastric fluid they will be capable enough to accomplish the criteria of an ideal floating drug delivery system which along with the maintenance of buoyancy for prolonged period of time allows the slow release of the drug from the dosage form which will be absorbed by the upper part of gastrointestinal tract which is the absorption site of drug. The duration of floating increases when low bulk density polymers were used. Parameters such as duration of floating and percentage floating capacity of various formulations can be seen from data given in table 5. Observed floating duration time was more than $15 \mathrm{~h}$ for all the prepared formulations. Experimental results clearly revealed that the floating duration time and \% floating capacity was directly related with the amount of polymers used. It may be due to the fact that after initial floating, it was the amount of swellable polymer that swelled and kept the density $<1$ which helped in providing buoyancy for longer periods of time.

Drug content of all the formulations except few was found to be sufficiently high. From the result of drug entrapment efficiency (table 5) it was found that all the prepared formulation entrapped more than $90 \%$ of drug (except $\mathrm{FM}_{7}$ ). It was observed that polymer concentration did not decide the fate of drug entrapment Optimisation of critical parameters such as mixing time, mixing speed at preformulation level plays a major role behind drug content and \% drug entrapment.

Table 5: Percentage Yield, drug content, drug entrapment and floating parameters of microsphere formulations $\mathrm{FM}_{1}$ to $\mathrm{FM}_{12}$

\begin{tabular}{|c|c|c|c|c|c|c|}
\hline \multirow{2}{*}{$\begin{array}{l}\text { Batch } \\
\text { code }\end{array}$} & \multicolumn{6}{|c|}{ Evaluation parameters mean \pm SD $(n=3)$} \\
\hline & Yield (\%) & $\begin{array}{l}\text { Theoretical drug content } \\
\text { (mg) }\end{array}$ & $\begin{array}{l}\text { Practical drug content } \\
\text { (mg) }\end{array}$ & $\begin{array}{l}\text { Drug entrapment } \\
(\%)\end{array}$ & $\begin{array}{l}\text { Floating duration } \\
\text { (h) }\end{array}$ & $\begin{array}{l}\text { \% floating } \\
\text { capacity }\end{array}$ \\
\hline $\mathrm{FM}_{1}$ & $80 \pm 2.43$ & $41.66 \pm 1.66$ & $40.8 \pm 0.55$ & $97.92 \pm 0.13$ & $17 \pm 1.6$ & $82 \pm 2$ \\
\hline $\mathrm{FM}_{2}$ & $72.5 \pm 1.78$ & $34.51 \pm 0.98$ & $33.1 \pm 0.91$ & $95.9 \pm 0.32$ & $15 \pm 1.1$ & $80 \pm 2$ \\
\hline $\mathrm{FM}_{3}$ & $70 \pm 3.59$ & $28.57 \pm 0.87$ & $27.77 \pm 0.76$ & $97.19 \pm 0.51$ & $16 \pm 1.8$ & $85 \pm 3$ \\
\hline $\mathrm{FM}_{4}$ & $66 \pm 2.19$ & $25 \pm 1.03$ & $23.12 \pm 0.87$ & $92.48 \pm 0.40$ & $15 \pm 2.1$ & $87 \pm 4$ \\
\hline $\mathrm{FM}_{5}$ & $82.85 \pm 2.67$ & $34.48 \pm 1.91$ & $32.1 \pm 0.79$ & $93.09 \pm 0.38$ & $15 \pm 2.3$ & $85 \pm 2$ \\
\hline $\mathrm{FM}_{6}$ & $66.66 \pm 3.13$ & $33.35 \pm 1.77$ & $33.02 \pm 0.35$ & $99 \pm 0.29$ & $17 \pm 1.3$ & $77 \pm 3$ \\
\hline $\mathrm{FM}_{7}$ & $76.66 \pm 1.03$ & $43.47 \pm 1.61$ & $36.52 \pm 0.56$ & $83.99 \pm 0.41$ & $19 \pm 0.7$ & $86 \pm 2$ \\
\hline $\mathrm{FM}_{8}$ & $72.5 \pm 1.45$ & $34.48 \pm 0.56$ & $33.6 \pm 0.41$ & $97.44 \pm 0.39$ & $20 \pm 0.3$ & $85 \pm 1$ \\
\hline $\mathrm{FM}_{9}$ & $85.23 \pm 2.09$ & $26.34 \pm 1.39$ & $25.98 \pm 0.32$ & $98.63 \pm 0.32$ & $22 \pm 0.3$ & $90 \pm 2$ \\
\hline $\mathrm{FM}_{10}$ & $73.33 \pm 2.17$ & $45.45 \pm 2.01$ & $43.22 \pm 0.82$ & $95.09 \pm 0.33$ & $18 \pm 1.2$ & $82 \pm 2$ \\
\hline $\mathrm{FM}_{11}$ & $72.5 \pm 3.41$ & $34.48 \pm 1.61$ & $31.72 \pm 0.53$ & $91.99 \pm 0.52$ & $18 \pm 1.5$ & $85 \pm 4$ \\
\hline $\mathrm{FM}_{12}$ & $75 \pm 2.77$ & $33.33 \pm 2.05$ & $32.92 \pm 0.40$ & $98.76 \pm 0.18$ & $17 \pm 1.2$ & $88 \pm 3$ \\
\hline
\end{tabular}

All the formulated microspheres shows good flow properties (table 6) with a value of the angle of repose between $21-29^{\circ}$.

The bulk density and the tapped density of all the formulations were within short range, i.e. $0.331 \mathrm{gm} / \mathrm{cm}^{3}$ to $0.572 \mathrm{gm} / \mathrm{cm}^{3}$ and 0.348 $\mathrm{gm} / \mathrm{cm}^{3}$ to $0.667 \mathrm{gm} / \mathrm{cm}^{3}$ respectively.
Carr's index of all the formulations was found to be less than 15 percent which indicates good flow properties. Also, Hausner's ratios of most of the formulations were found to be less than 1.25 which indicates better flow properties. Excellent flow properties of prepared floating microsphere suggested less polydispersity, complete drying and particle size uniformity.

Table 6: Rheological characteristics of microsphere formulations $\mathrm{FM}_{1}$ to $\mathrm{FM}_{12}$

\begin{tabular}{|c|c|c|c|c|c|}
\hline \multirow{2}{*}{$\begin{array}{l}\text { Batch } \\
\text { code }\end{array}$} & \multicolumn{5}{|c|}{ Evaluation of rheological properties mean $\pm S D(n=3)$} \\
\hline & Angle of repose $\left(^{\circ}\right)$ & $\begin{array}{l}\text { Bulk density } \\
\left(\mathrm{g} / \mathrm{cm}^{3}\right)\end{array}$ & $\begin{array}{l}\text { Tapped density } \\
\left(\mathrm{g} / \mathrm{cm}^{3}\right)\end{array}$ & Carr's index & Hausner's ratio \\
\hline $\mathrm{FM}_{1}$ & $24.33 \pm 0.05$ & $0.444 \pm 0.01$ & $0.487 \pm 0.02$ & $8.829 \pm 0.67$ & $1.096 \pm 0.56$ \\
\hline $\mathrm{FM}_{2}$ & $21.32 \pm 0.03$ & $0.408 \pm 0.01$ & $0.465 \pm 0.03$ & $12.25 \pm 0.58$ & $1.139 \pm 0.34$ \\
\hline $\mathrm{FM}_{3}$ & $27.54 \pm 0.05$ & $0.416 \pm 0.02$ & $0.487 \pm 0.01$ & $14.57 \pm 0.43$ & $1.170 \pm 0.51$ \\
\hline $\mathrm{FM}_{4}$ & $24.32 \pm 0.1$ & $0.350 \pm 0.01$ & $0.390 \pm 0.01$ & $10.25 \pm 0.19$ & $1.114 \pm 0.13$ \\
\hline $\mathrm{FM}_{5}$ & $29.73 \pm 0.02$ & $0.400 \pm 0.01$ & $0.465 \pm 0.01$ & $13.97 \pm 0.13$ & $1.162 \pm 0.09$ \\
\hline $\mathrm{FM}_{6}$ & $26.46 \pm 0.09$ & $0.331 \pm 0.01$ & $0.348 \pm 0.01$ & $4.060 \pm 0.14$ & $1.042 \pm 0.14$ \\
\hline $\mathrm{FM}_{7}$ & $25.29 \pm 0.09$ & $0.400 \pm 0.02$ & $0.444 \pm 0.02$ & $9.909 \pm 0.21$ & $1.110 \pm 0.11$ \\
\hline $\mathrm{FM}_{8}$ & $27.89 \pm 0.07$ & $0.400 \pm 0.02$ & $0.425 \pm 0.02$ & $5.882 \pm 0.25$ & $1.062 \pm 0.14$ \\
\hline $\mathrm{FM}_{9}$ & $29.54 \pm 0.05$ & $0.572 \pm 0.02$ & $0.667 \pm 0.01$ & $14.26 \pm 0.19$ & $1.166 \pm 0.21$ \\
\hline $\mathrm{FM}_{10}$ & $24.82 \pm 0.05$ & $0.416 \pm 0.01$ & $0.487 \pm 0.01$ & $14.57 \pm 0.22$ & $1.170 \pm 0.27$ \\
\hline $\mathrm{FM}_{11}$ & $25.04 \pm 0.05$ & $0.425 \pm 0.01$ & $0.487 \pm 0.01$ & $12.73 \pm 0.28$ & $1.145 \pm 0.19$ \\
\hline $\mathrm{FM}_{12}$ & $21.05 \pm 0.05$ & $0.454 \pm 00.01$ & $0.512 \pm 0.01$ & $11.32 \pm 0.11$ & $1.127 \pm 0.15$ \\
\hline
\end{tabular}


Loose surface crystal studies were done to know the excess of drug present on the surface of microspheres. These studies play an important role in the determination of drug release profile because sometimes due to the adherence of crystals of the drug on the surface of microspheres they give a false alarm of burst effect which interferes in the correct determination of release profile of drug from the dosage form. Data given in the table number 7 clearly indicates that there is little or no adherence of drug on the surface of microspheres. Less surface entrapment signified a large amount of drug was entrapped inside the polymeric matrix. It may also conclude from the observed result that less surface drug entrapment reduced the chances of burst release which provided more controlled release of the drug.

Table 7: Loose surface crystal studies of formulations $\mathrm{FM}_{1}$ to $\mathrm{FM}_{12}$

\begin{tabular}{|c|c|c|c|}
\hline \multirow[t]{2}{*}{ Batch Code } & \multicolumn{3}{|c|}{ Loose surface crystal study mean \pm SD $(n=3)$} \\
\hline & Drug content in filtrate & Loaded dug content(mg) & $\%$ Total drug content \\
\hline $\mathrm{FM}_{1}$ & 1.519 & 41.66 & $3.646 \pm 0.69$ \\
\hline $\mathrm{FM}_{2}$ & 1.213 & 34.51 & $3.514 \pm 0.91$ \\
\hline $\mathrm{FM}_{3}$ & 1.131 & 28.57 & $3.958 \pm 0.73$ \\
\hline $\mathrm{FM}_{4}$ & 0.862 & 25 & $3.448 \pm 0.94$ \\
\hline $\mathrm{FM}_{5}$ & 2.912 & 34.48 & $8.445 \pm 0.09$ \\
\hline $\mathrm{FM}_{6}$ & 1.537 & 33.35 & $4.608 \pm 0.18$ \\
\hline $\mathrm{FM}_{7}$ & 1.321 & 43.478 & $3.038 \pm 0.34$ \\
\hline $\mathrm{FM}_{8}$ & 1.269 & 34.48 & $3.680 \pm 0.51$ \\
\hline $\mathrm{FM}_{9}$ & 0.431 & 26.34 & $1.598 \pm 0.69$ \\
\hline $\mathrm{FM}_{10}$ & 1.152 & 45.45 & $2.534 \pm 0.71$ \\
\hline $\mathrm{FM}_{11}$ & 0.902 & 34.48 & $2.616 \pm 0.39$ \\
\hline $\mathrm{FM}_{12}$ & 0.792 & 33.33 & $2.372 \pm 0.56$ \\
\hline
\end{tabular}

One of the important characteristic to know the release of drug from the dosage form is the swelling behaviour. As greater the swelling greater will be the release of drug from the microspheres. Formulations prepared with ethyl cellulose showed no significant increase in swelling behaviour as a number of polymer increases which can be seen from the data given in table number 8 . All the formulations showed about 14 to
15 percent increase in swelling for three hours. Formulations of HPMC showed about 14 to 18 percent of swelling for same time period, and formulations of ethyl cellulose and HPMC showed about17 to 25 percent of swelling for same time period. \% Swelling was depends on the water uptake characteristics of the polymer as well as their polymeric chain relaxation capability. Thus the observed values were different from each other.

Table 8: Swelling index of formulations $\mathrm{FM}_{1}$ to $\mathrm{FM}_{12}$

\begin{tabular}{llll}
\hline Batch code & Initial weight $(\mathbf{m g})$ & Final weight $(\mathbf{m g})$ & Mean $\%$ swelling of microspheres \pm SD (n=3) \\
\hline $\mathrm{FM}_{1}$ & 100 & 114.5 & $14.5 \pm 1.33$ \\
$\mathrm{FM}_{2}$ & 100 & 115.3 & $15.3 \pm 1.29$ \\
$\mathrm{FM}_{3}$ & 100 & 115.8 & $15.8 \pm 1.11$ \\
$\mathrm{FM}_{4}$ & 100 & 114.7 & $14.7 \pm 1.21$ \\
$\mathrm{FM}_{5}$ & 100 & 115.3 & $15.3 \pm 1.49$ \\
$\mathrm{FM}_{6}$ & 100 & 114.9 & $14.9 \pm 1.51$ \\
$\mathrm{FM}_{7}$ & 100 & 118.8 & $18.8 \pm 1.80$ \\
$\mathrm{FM}$ & 118.1 & $18.1 \pm 1.72$ \\
$\mathrm{FM}_{9}$ & 100 & 117.2 & $17.2 \pm 1.23$ \\
$\mathrm{FM}_{10}$ & 100 & 125.8 & $25.8 \pm 1.41$ \\
$\mathrm{FM}_{11}$ & 100 & 125.5 & $25.5 \pm 1.32$ \\
$\mathrm{FM}_{12}$ & 100 & 125.3 & $25.3 \pm 1.88$ \\
\hline
\end{tabular}

In vitro release of ciprofloxacin from different formulations were studied in $0.1 \mathrm{~N} \mathrm{HCl}$ for $10 \mathrm{~h}$ using USP-II type dissolution apparatus. From the release data of different formulations it has been observed that the cumulative percentage release of drug from the microspheres depends upon the type and amount of polymer used. The release data of dosage form given in the table number 9 and 10 revealed the fact that the combination of polymers also affects the release rate. For the formulation $\mathrm{FM}_{1}$ to $\mathrm{FM}_{4}$ ethyl cellulose was used in gradually increasing concentrations with constant drug concentration. Important characteristics of ethylcellulose such as its non-toxicity, non-allergic and nonirritant behaviour make this polymer one of the best for the formulation of microspheres. From the present study, it was observed that the formulations prepared from this polymer possess lower densities (having a hollow core), exhibit higher buoyancy and are expected to be retained in the gastric environment for a prolonged period of time. Most of the formulations prepared from ethyl cellulose did not show any burst effect or lag time which indicates homogeneous drug distribution. The release of ciprofloxacin significantly decreased with increasing amount of ethyl cellulose because when the higher concentration of polymer has used the density of polymer matrix gets increased due to the formation of the strong polymeric network which increases the diffusional path length. Thus the drug release from the polymer matrix gets reduced. HPMC was selected for the preparation of formulations $\mathrm{FM}_{5}$ to $\mathrm{FM}_{8}$ because it is known to be soluble at $\mathrm{pH}>5.5$ and has low solubility in acidic medium. These characteristics of HPMC help the dosage form to control the release rate of a medicament for desired period of time so that it can meet the criteria of ideal floating dosage form. Also, more than $90 \%$ of microspheres prepared from HPMC maintained the floating duration for 15 to $24 \mathrm{~h}$ of a longer period of time and those microspheres which lost their buoyancy might successfully pass through the stomach and release the drug in significant amount in the upper part of gastrointestinal tract the absorption site of the drug.

Formulations $\mathrm{FM}_{9}$ to $\mathrm{FM}_{12}$ were prepared using ethyl cellulose and hydroxypropyl methylcellulose (HPMC). Formulation $\mathrm{FM}_{9}$ shows $29.56 \%$ of cumulative drug release at first hour and $\mathrm{FM}_{11}$ shows $16.403 \%$ of cumulative drug release at first hour. The rate of $\mathrm{FF}_{11}$ formulation is very much controlled because of the combination of polymers. When both the polymers are combined, they form a polymeric matrix of high density due to the formation of the strong polymeric network. When the concentration of ethylcellulose is increased as in $\mathrm{FF}_{11}$ (drug: ethylcellulose: HPMC 1:2:1) compared to $\mathrm{FM}_{9}$ (drug: ethyl- 
cellulose: HPMC 1:1:1) the cumulative release of ciprofloxacin significantly decreased because increase in concentration of ethylcellulose increases the density of polymer matrix which in turns increases the diffusional path length. The release of $\mathrm{FM}_{9}$ and $\mathrm{FM}_{10}$ is higher than that of $\mathrm{FM}_{11}$ because at lower polymer concentration smaller microspheres are formed; due to the small size of microspheres larger surface area is exposed to dissolution medium giving rise to faster drug release. Formulation $\mathrm{FM}_{12}$ (drug: ethylcellulose: HPMC 2:1:1) shows $51.48 \%$ drug release at first hour due to improper drug and polymer ratio.

Table 9: In vitro drug release profile of formulations $F_{1}$ to $F_{6}$

\begin{tabular}{|c|c|c|c|c|c|c|}
\hline \multirow[t]{2}{*}{ Time (h) } & \multicolumn{6}{|c|}{$\%$ cumulative amount of drug release $(n=3)$ mean \pm SD } \\
\hline & $\mathbf{F M}_{1}$ & $\mathbf{F M}_{2}$ & $\mathbf{F M}_{3}$ & $\mathbf{F M}_{4}$ & $\mathbf{F M}_{5}$ & $\mathbf{F M}_{6}$ \\
\hline $\mathbf{0}$ & $\mathbf{0}$ & $\mathbf{0}$ & $\mathbf{0}$ & $\mathbf{0}$ & $\mathbf{0}$ & $\mathbf{0}$ \\
\hline 1 & $34.41 \pm 1.18$ & $32.084 \pm 1.38$ & $26.93 \pm 1.06$ & $20.242 \pm 0.93$ & $28.59 \pm 1.24$ & $27.256 \pm 0.95$ \\
\hline 2 & $40.77 \pm 1.23$ & $43.138 \pm 1.51$ & $29.686 \pm 1.32$ & $23.468 \pm 1.97$ & $36.439 \pm 0.99$ & $32.647 \pm 0.55$ \\
\hline 3 & $38.35 \pm 1.45$ & $40.114 \pm 1.90$ & $32.456 \pm 1.48$ & $27.491 \pm 1.35$ & $36.61 \pm 1.89$ & $39.051 \pm 1.36$ \\
\hline 4 & $42.98 \pm 0.13$ & $41.422 \pm 1.29$ & $39.584 \pm 1.27$ & $33.092 \pm 0.89$ & $45.01 \pm 1.23$ & $49.321 \pm 1.27$ \\
\hline 5 & $43.21 \pm 1.00$ & $42.193 \pm 1.62$ & $43.277 \pm 1.91$ & $33.274 \pm 0.94$ & $45.89 \pm 0.57$ & $54.561 \pm 0.96$ \\
\hline 6 & $43.89 \pm 1.23$ & $38.616 \pm 1.83$ & $47.857 \pm 1.32$ & $35.012 \pm 1.37$ & $46.844 \pm 1.41$ & $59.342 \pm 1.54$ \\
\hline 7 & $43.24 \pm 1.14$ & $38.824 \pm 1.22$ & $48.986 \pm 1.25$ & $40.653 \pm 1.59$ & $52.728 \pm 1.07$ & $64.842 \pm 0.85$ \\
\hline 8 & $43.48 \pm 1.62$ & $42.296 \pm 1.76$ & $52.726 \pm 1.53$ & $47.102 \pm 1.28$ & $58.72 \pm 1.12$ & $67.594 \pm 0.49$ \\
\hline 9 & $45.29 \pm 0.99$ & $43.715 \pm 1.54$ & $58.223 \pm 1.87$ & $51.25 \pm 0.79$ & $68.059 \pm 0.38$ & $69.067 \pm 1.33$ \\
\hline 10 & $47.36 \pm 0.82$ & $45.039 \pm 1.39$ & $60.926 \pm 1.31$ & $54.641 \pm 0.81$ & $72.700 \pm 0.75$ & $72.491 \pm 0.59$ \\
\hline
\end{tabular}

Table 10: In vitro drug release profile of formulations $\mathrm{FM}_{7}$ to $\mathrm{FM}_{12}$

\begin{tabular}{|c|c|c|c|c|c|c|}
\hline \multirow[t]{2}{*}{ Time (h) } & \multicolumn{6}{|c|}{$\%$ cumulative amount of drug release $(n=3)$ mean \pm SD } \\
\hline & $\mathbf{F M}_{7}$ & $\mathbf{F M}_{8}$ & FMa & $\mathbf{F M}_{10}$ & $\mathbf{F M}_{11}$ & $\mathbf{F M}_{12}$ \\
\hline $\mathbf{0}$ & $\mathbf{0}$ & $\mathbf{0}$ & $\mathbf{0}$ & $\mathbf{0}$ & $\mathbf{0}$ & $\mathbf{0}$ \\
\hline 1 & $49.82 \pm 1.12$ & $12.631 \pm 0.76$ & $29.56 \pm 0.49$ & $34.615 \pm 1.93$ & $16.403 \pm 0.63$ & $51.48 \pm 1.94$ \\
\hline 2 & $68.84 \pm 1.83$ & $14.807 \pm 0.98$ & $30.15 \pm 0.81$ & $34.807 \pm 1.99$ & $17.588 \pm 0.87$ & $58.12 \pm 1.89$ \\
\hline 3 & $69.78 \pm 0.97$ & $15.084 \pm 1.71$ & $35.73 \pm 0.94$ & $35.000 \pm 1.78$ & $19.325 \pm 0.59$ & $58.44 \pm 1.49$ \\
\hline 4 & $72.29 \pm 0.27$ & $17.982 \pm 1.27$ & $36.76 \pm 1.13$ & $36.327 \pm 1.26$ & $22.165 \pm 0.92$ & $59.43 \pm 1.62$ \\
\hline 5 & $73.22 \pm 1.37$ & $18.573 \pm 1.03$ & $37.37 \pm 0.73$ & $41.633 \pm 1.06$ & $22.834 \pm 1.34$ & $60.99 \pm 1.39$ \\
\hline 6 & $74.157 \pm 1.93$ & $20.259 \pm 1.47$ & $40.49 \pm 0.28$ & $41.86 \pm 0.63$ & $27.332 \pm 1.95$ & $61.33 \pm 1.93$ \\
\hline 7 & $74.559 \pm 1.80$ & $26.342 \pm 0.91$ & $43.41 \pm 1.03$ & $45.491 \pm 0.71$ & $29.668 \pm 1.27$ & $62.93 \pm 1.28$ \\
\hline 8 & $72.28 \pm 0.75$ & $32.801 \pm 0.77$ & $44.28 \pm 0.32$ & $44.602 \pm 1.83$ & $31.47 \pm 0.25$ & $69.62 \pm 1.11$ \\
\hline 9 & $76.41 \pm 0.69$ & $34.637 \pm 1.87$ & $49.51 \pm 0.27$ & $52.559 \pm 1.44$ & $34.921 \pm 0.58$ & $70.00 \pm 1.51$ \\
\hline 10 & $74.6 \pm 1.91$ & $53.938 \pm 1.29$ & $60.13 \pm 0.92$ & $55.34 \pm 1.01$ & $36.749 \pm 0.22$ & $73.55 \pm 1.79$ \\
\hline
\end{tabular}

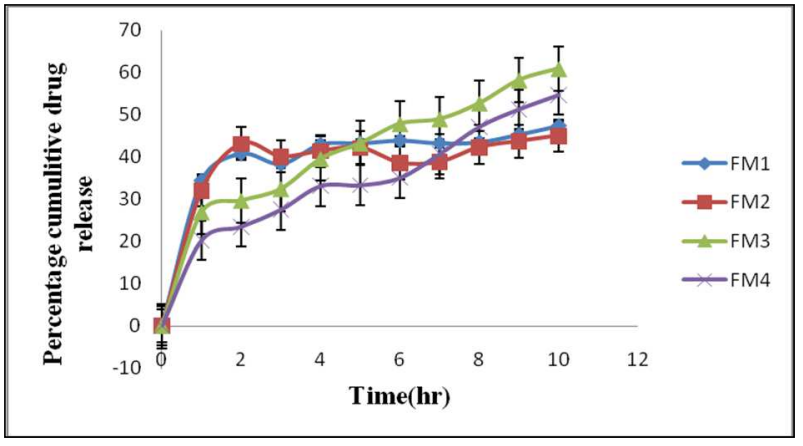

Fig. 3: In vitro dissolution plots of formulation $\mathrm{FM}_{1}-\mathrm{FM}_{4}$

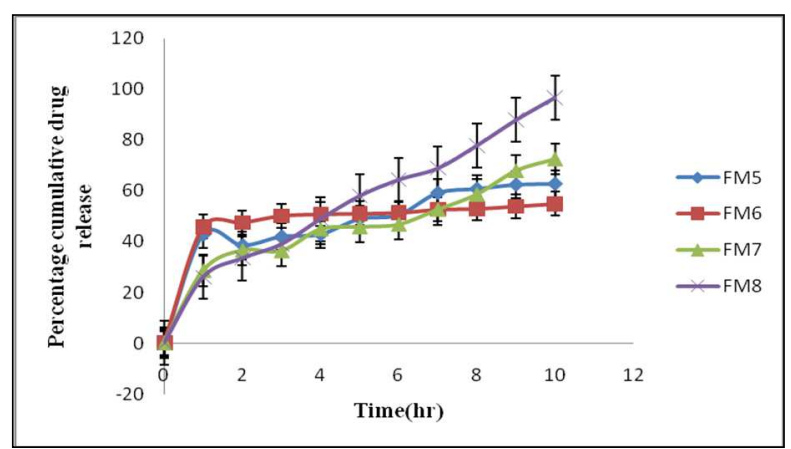

Fig. 4: In vitro dissolution plots of formulation $\mathrm{FM}_{5}-\mathrm{FM}_{8}$

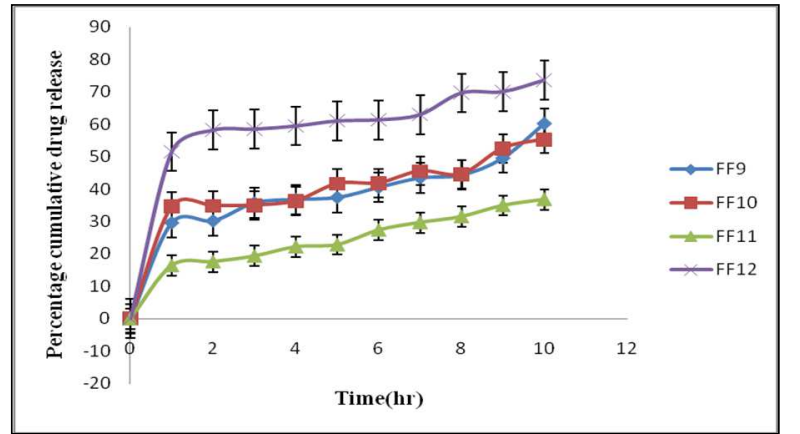

Fig. 5: In vitro dissolution plots of formulation $\mathrm{FM}_{9}-\mathrm{FM}_{12}$

In order to establish the mechanism of release of the drug from the immediate and modified release, dosages forms kinetic models are used. The drug release data were subjected to various mathematical kinetic models like zero order, first order, etc. The data were also subjected to Higuchi's model and Korsmeyer model. Korsmeyer model is widely used; when the release mechanism is not well known or when more than one type of release phenomena could be involved. Korsmeyer and Peppas equation: $\mathrm{Mt}_{\mathrm{t}} \mathrm{M}_{\propto}=\mathrm{Kt}^{\mathrm{n}}$, where $M t / M_{\propto}$ is the fractional drug release in time' $t$ '. $K=$ constant incorporating of structural and geometric characteristics of the controlled release device, $\mathrm{n}=$ diffusional release exponent indicative of release mechanism. The ' $n$ ' value could be used to characterize different release mechanisms as follows $n=0.5$ means Fickian diffusion, $0.5<n<1.0$ non-Fickian diffusion, and $n=1.0$ Case II diffusion [14]. The interpretation of data was based on the value of the resulting regression coefficients. 
For all the formulations the values of $\mathrm{R}^{2}$ of zero order, first order and Higuchi were given in table number 11 and from this table it was clearly observed that for most of the formulations the value of resulting regression coefficient $\left(\mathrm{R}^{2}\right)$ is highest for Higuchi model which shows all the formulations predominantly followed Higuchi square root kinetics indicating a diffusion dependent release as expected from a matrix system like the microspheres. The corresponding $\mathrm{n}$ values of the maximum formulation were below 0.45 which indicates that the formulations released the drug through Fickian diffusion mechanism.

Table 11: Result of regression coefficients of release data by curve fitting method on zero-order, first-order and Higuchi kinetic model and their diffusion exponent $(n)^{*}$

\begin{tabular}{llll}
\hline Batch code & regression coefficients $\left(\mathbf{r}^{2}\right)$ & & Higuchi \\
\cline { 2 - 3 } & Zero-order & First-order & 0.952 \\
$\mathrm{FM}_{1}$ & 0.705 & 0.448 & 0.114 \\
$\mathrm{FM}_{2}$ & 0.621 & 0.620 & 0.915 \\
$\mathrm{FM}_{3}$ & 0.934 & 0.953 & 0.973 \\
$\mathrm{FM}_{4}$ & 0.930 & 0.951 & 0.983 \\
$\mathrm{FM}_{5}$ & 0.797 & 0.862 & 0.916 \\
$\mathrm{FM}_{6}$ & 0.652 & 0.698 & 0.938 \\
$\mathrm{FM}_{7}$ & 0.719 & 0.636 & 0.376 \\
$\mathrm{FM}_{8}$ & 0.972 & 0.820 & 0.724 \\
$\mathrm{FM}_{9}$ & 0.811 & 0.840 & 0.971 \\
$\mathrm{FM}_{10}$ & 0.762 & 0.813 & 0.150 \\
$\mathrm{FM}_{12}$ & 0.918 & 0.927 & 0.517 \\
\end{tabular}

$* \mathrm{Mt} / \mathrm{M} \infty=\mathrm{kt}^{\mathrm{n}}$

On the basis of all the evaluation parameters formulations $\mathrm{FM}_{9}$ was selected as final formulations, and its statistical evaluation was done using analysis of variance (ANOVA) at $\mathrm{P} \leq 0.05$ significance level and it was found to be statistically significant with $P$ value $<0.05$.
The compatibility study was performed by means of FTIR instrument. The result was based on matching the main peak of the pure drug with the formulation, as the peak table (table number 12) shows there was no interaction between ciprofloxacin hydrochloride and polymer.

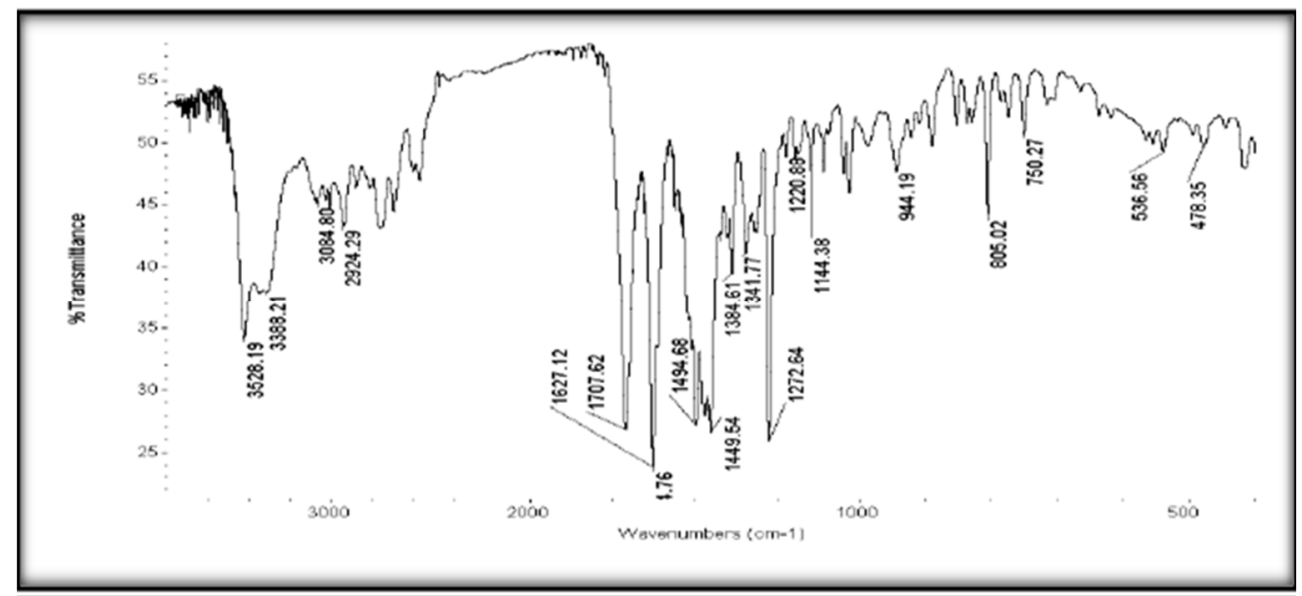

Fig. 6: FTIR spectra of ciprofloxacin hydrochloride

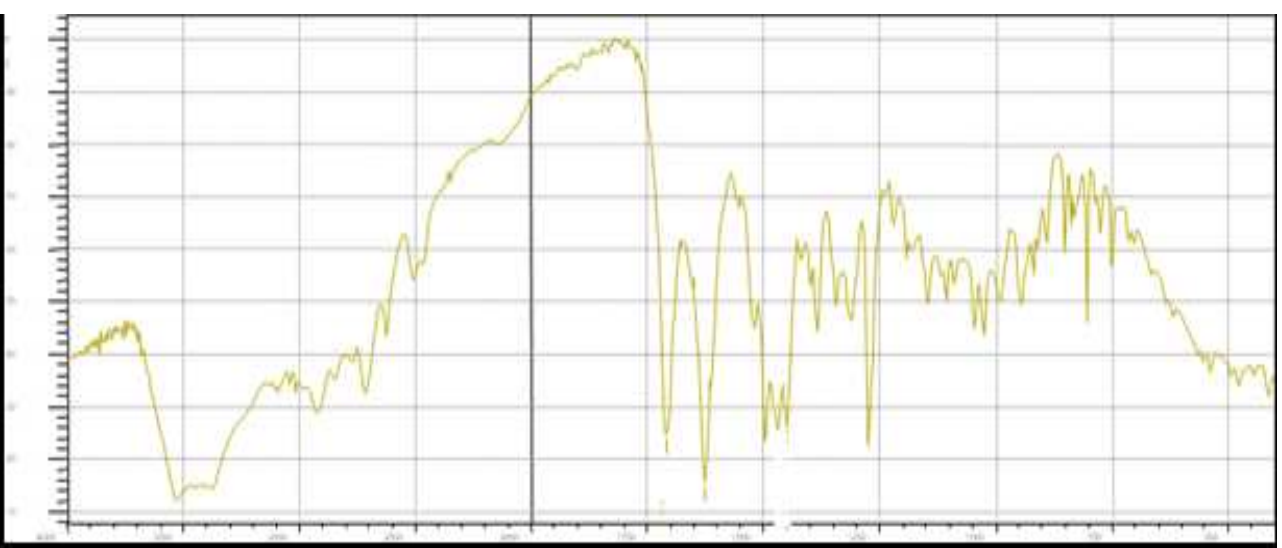

Fig. 7: FTIR spectra of FMg formulation 
Table 12: FTIR peak table

\begin{tabular}{lll}
\hline Drug peak & Formulation peak & Peak characteristics \\
\hline 3288.21 & 3285.20 & - OH stretch \\
1710.6 & 1707.60 & - COOH stretch \\
1627.01 & 1627.00 & $-\mathrm{C}==0$ vibration \\
1498.84 & 1496.50 & C-H stretch \\
1384.81 & 1381.13 & Aromatic C=C \\
1272.64 & 1271.20 & C-F stretch \\
\hline
\end{tabular}

\section{CONCLUSION}

Floating micro-particles of ciprofloxacin were successfully developed using polymers such as ethyl cellulose and HPMC. Results obtained shows that ciprofloxacin floating microspheres increases the gastric residence time as well as bioavailability and simultaneously decrease the dosing interval and dosing amount. It can be concluded that the developed floating microspheres of ciprofloxacin can be used for prolonged drug release in the stomach for at least $8 \mathrm{~h}$, thereby improving the bioavailability and patient compliance.

\section{CONFLICT OF INTERESTS}

Declare none

\section{REFERENCES}

1. Klausner EA, Lavy E, Friedman M, Hoffman A. Expandable gastro-retentive dosage forms. J Controlled Release 2003;90:143-62.

2. Gutierrez-Rocca J, Omidian H, Shah K. Progresses in gastro retentive drug delivery systems. PharmaTech; 2003. p. 152-6.

3. Desai KG, Park HJ. Study of gamma-irradiation effects on chitosan microparticles. Drug Delivery 2006;13:39-50.

4. Patel A, Ray S, Thakur RS. In vitro evaluation and optimisation of controlled release floating drug delivery system of metformin hydrochloride. Daru 2006;14:57-64.

5. Shabaraya AR, Narayanacharyulu R. Design and evaluation of chitosan microspheres of metoprolol tartrate for sustained release. Indian J Pharm Sci 2003;65:250-2.

6. Jain SK, Agrawal GP, Jain NK. Evaluation of porous carrierbased is floating orlistat microspheres for gastric delivery. AAPS Pharm Sci Tech 2006;7:E1-E9.

7. Rahman Z, Ali M, Khar RK. Design and evaluation of bilayer floating tablets of captopril. Acta Pharm 2006;56:49-57.
8. Levis SR, Deasy P. Pharmaceutical applications of size reduced grades of surfactant co-processed microcrystalline cellulose. Int J Pharm 2001;230:25-33.

9. Chowdary KPR, Rao YS. Mucoadhesive microcapsules of glipizide: characterization, in vitro and in vivo evaluation. Indian J Pharm Sci 2003;65:279-84.

10. Sinko PJ, Singh Y, Martin AN. Micromeritics Martin's physical pharmacy and pharmaceutical sciences: physical chemical and biopharmaceutical principles in the pharmaceutical sciences. Philadelphia: Lippincott Williams Wilkins 2006;6:442-68.

11. Kulkarni GT, Gowthamarajan K, Suresh B. Stability testing of pharmaceutical products: an overview. Indian J Pharm Educ 2004;38:194-202.

12. Shovarani KN, Goundalkar AG. Preparation and evaluation of microsphere of diclofenac sodium. Indian J Pharm Sci 1994;56:45-50.

13. Bhumkar DR, Maheshwari M, Patil VB, Pokharkar VB. Studies on the effect of variabilities by response surface methodology for naproxane microspheres. Indian Drugs 2003;40:455-61.

14. Velivela S, Abbulu K, Vinyas M, Pati NB. Formulation and in vitro evaluation of ritonavir floating tablets by melt granulation technique. Int J Appl Pharm 2016;8:12-5.

15. Sakhare SS, Yadav AV, Jadhav PD. Design, development and characterization of mucoadhesive gastro spheres of carvedilol. Int J Appl Pharm 2016;8:37-42.

16. Peppas NA. Analysis of fickian and non-fickian drug release from polymers. Pharm Acta Helv 1985;60:110-1.

\section{How to cite this article}

- Sumit Durgapal, Sayantan Mukhopadhyay, Laxmi Goswami. Preparation, characterization and evaluation of floating microparticles of ciprofloxacin. Int J Appl Pha 2017;9(1):1-8. 